\title{
Location of Triple-Negative Breast Cancers: Comparison with Estrogen Receptor-Positive Breast Cancers on MR Imaging
}

\author{
Won Hwa Kim ${ }^{1}$, Wonshik Han ${ }^{2}$, Jung Min Chang ${ }^{1}$, Nariya Cho ${ }^{1}$, In Ae Park ${ }^{3}$, Woo \\ Kyung Moon ${ }^{1}$ *
}

1 Department of Radiology, Seoul National University Hospital, Seoul, Republic of Korea, 2 Department of Surgery, Seoul National University College of Medicine, Seoul, Korea, 3 Department of Pathology, Seoul National University College of Medicine, Seoul, Korea

*moonwk@snu.ac.kr

\section{G open access}

Citation: Kim WH, Han W, Chang JM, Cho N, Park IA, Moon WK (2015) Location of Triple-Negative Breast Cancers: Comparison with Estrogen Receptor-Positive Breast Cancers on MR Imaging. PLoS ONE 10(1): e0116344. doi:10.1371/journal. pone. 0116344

Academic Editor: Kenneth G.A. Gilhuijs, University Medical Centre Utrecht, NETHERLANDS

Received: September 2, 2014

Accepted: December 8, 2014

Published: January 21, 2015

Copyright: @ $2015 \mathrm{Kim}$ et al. This is an open access article distributed under the terms of the Creative Commons Attribution License, which permits unrestricted use, distribution, and reproduction in any medium, provided the original author and source are credited.

Data Availability Statement: All raw data files are available from DRYAD (accession numbers, http://doi. org/10.5061/dryad.32765).

Funding: This work was supported by the National Research Foundation of Korea (NRF) grant funded by the Korean government (MEST) (No.

2012R1A2A1A01010846). The funders had no role in study design, data collection and analysis, decision to publish, or preparation of the manuscript.

Competing Interests: The authors have declared that no competing interests exist.

\section{Abstract}

There has been a major need to better understand the biological characteristics of triplenegative breast cancers. Compared with estrogen receptor (ER)-positive cancers, several magnetic resonance (MR) imaging findings have been reported as characteristic findings. However, information regarding their location has not been described. Our study was to compare the location of triple-negative breast cancers with that of ER-positive breast cancers using magnetic resonance (MR) imaging. The locations of 1102 primary breast cancers (256 triple-negative and 846 ER-positive) in 1090 women (mean, 52.1 years) were reviewed using three-dimensional $(3 \mathrm{D})$ coordinates. The $\mathrm{x}$-axis measurement was recorded as the transverse distance from the posterior nipple line; $y$-axis measurement as the anteroposterior distance from the chest wall; z-axis measurement as the superoinferior distance from the posterior nipple line. The association between breast cancer subtype and tumor location was evaluated using multiple linear regression analysis. Triple-negative breast cancers were significantly closer to the chest wall than ER-positive breast cancers in absolute $(1.8 \mathrm{~cm}$ vs. $2.3 \mathrm{~cm}, P<.0001)$ and normalized $(0.21$ vs. $0.25, P<.0001) \mathrm{y}$-axis distances. The $\mathrm{x}$ - and $\mathrm{z}$-axes distances were not significantly different between triple-negative and ER-positive breast cancers. Multiple linear regression analysis revealed that age, mammographic density, axillary nodal status, and triple-negative subtype were significantly associated with absolute and normalized distances from the chest wall (all $P<.05$ ). Our results show that triple-negative breast cancers have a tendency toward a posterior or prepectoral location compared with ER-positive breast cancers.

\section{Introduction}

Previous studies have shown that the location of a primary cancer could affect tumor growth patterns and have a prognostic significance [1]. One such study reported that patients with tumors in the medial location of the breast have worse prognosis $[2,3]$, and occult spread to 
internal mammary lymph nodes was attributed to an increased risk of relapse and breast cancer death for patients with tumors in the medial location of the breast. Moreover, research has demonstrated that lymph node metastasis occurs more commonly in tumors located in the lateral portion of the breast $[4,5]$. In addition to their prognostic significance, familial breast cancers have been reported to have a preferential location. One study showed that more than half (42 of 75) of their sample of familial breast cancers resided in the posterior region of the breast, and most frequently ( 40 of 42 cancers) the immediate prepectoral region of the breast [6]. Furthermore, a posterior or prepectoral tumor location has been regarded as a reason for missed cancers on mammography, particularly when combined with dense parenchyma [7-9].

Recently, there has been a major need to better understand the biological characteristics of triple-negative (estrogen receptor [ER]-negative, progesterone receptor [PR]-negative, and human epidermal growth factor receptor 2 [HER2]-negative) breast cancer, which accounts for $15 \%$ to $20 \%$ of newly diagnosed breast cancer cases [10-12]. Triple-negative breast cancers have been reported to have less axillary lymph node metastasis but poorer prognosis due to distant metastasis compared with ER-positive cancers $[11,13]$. The imaging and histopathologic features of triple-negative breast cancers have been described in the literature [14]. Compared with ER-positive cancers, several magnetic resonance (MR) imaging findings, such as smooth margin, rim enhancement, and intratumoral necrosis have been reported as characteristic findings [14-16]. However, information regarding their location, to our knowledge, has not been described. Our hypothesis was that triple-negative breast cancers, the most aggressive and common type of breast cancer in younger women with a family history, may have a tendency to be located in the medial or posterior region of the breast. Anatomical and lymphoscintigraphic studies have revealed that tumors in the medial and posterior locations have considerable lymphatic dissemination to the internal mammary node chain, which is the most important destination for lymph drainage outside of the axilla [17].

Thus, the aim of this study was to compare the location of triple-negative breast cancers with that of ER-positive breast cancers using MR imaging.

\section{Materials and Methods}

\section{Patients}

Institutional review board of Seoul National University Hospital approved our retrospective study and the requirement for informed consent was waived for this retrospective analysis. Patient record or information was anonymized and de-identified prior to analysis. Between June 2009 and May 2012, a search of a computerized MR imaging and pathology database identified 411 patients with triple-negative breast cancers and 1244 patients with ER-positive breast cancers who were diagnosed with invasive breast cancer and underwent breast MR imaging prior to surgery at our institution. HER2-enriched cancers were excluded to focus on comparing the most contrasting subtypes (triple-negative and ER-positive cancers) and simplify into two comparison groups to minimize potential statistical errors from multiple comparison. ERnegative, PR-positive, and HER2-negative cancers were also excluded for the same reasons. Among these 1655 patients, patients who received neoadjuvant chemotherapy and without pre-therapy MR imaging $(\mathrm{n}=114)$, patients who underwent excisional biopsy or had prior breast surgery before MR imaging $(\mathrm{n}=79)$, and those without immunohistochemistry (IHC) or fluorescence in situ hybridization (FISH) for HER2 $(\mathrm{n}=15)$ were excluded. Patients with microinvasive breast cancers $(n=15)$, multifocal or multicentric breast cancers $(n=336)$, and those with breast implants $(n=6)$ were also excluded due to difficulty in determining the exact location of the invasive cancer. Finally, 1102 primary breast cancers (256 triple-negative and 846 ER-positive) in 1090 patients (bilateral cancers in 12 patients) comprised our study group. 
The mean age of the patients was 52.1 years (range, 20-84 years). A total of 432 cancers (39.2\%) were detected at screening. The mean size of the tumors was $2.5 \mathrm{~cm}$ (range, $0.2 \mathrm{~cm}-5.9 \mathrm{~cm}$ ). A total of 924 of the 1102 cancers (83.8\%) were treated with breast-conserving surgery, and 178 cancers (16.2\%) were treated with mastectomy. During this period, MR imaging was routinely performed in all patients prior to surgery for breast cancer in our institution.

\section{MR imaging examination}

MR imaging was performed with the patient placed in a prone position. MR examinations were performed using a 1.5-T scanner (Signa; General Electric Medial Systems, Milwaukee, WI) with a dedicated breast coil (8-channel HD breast array, General Electric Medical Systems). After obtaining a bilateral transverse localizer image, sagittal fat-suppressed T2-weighted fast spin-echo images were obtained (TR/TE, variable from 5500 to $7150 / 82 ; 256 \times 160$ matrix; field of view, $200 \times 200 \mathrm{~mm}$; 1.5 -mm slice thickness, no gap). Dynamic contrast-enhanced examinations included one pre-contrast and five post-contrast bilateral sagittal image acquisitions using a fat-suppressed T1-weighted three-dimensional (3D) fast spoiled gradient echo sequence (TR/TE, $6.5 / 2.5 ; 256 \times 160$ matrix; flip angle, $10^{\circ}$; field of view, $200 \times 200 \mathrm{~mm} ; 1.5-\mathrm{mm}$ slice thickness, no gap). Gadobenate dimeglumine $(0.1 \mathrm{mmol} / \mathrm{kg}$ Multihance; Bracco Imaging, Milan, Italy) was injected using an automated injector (Spectris MR, Medrad Europe, Maastricht, Netherlands) through an indwelling IV catheter. Five post-contrast image series were obtained at 76, 165, 345, 434, and 583 seconds after contrast administration. For all studies, early subtraction (i.e., first post-contrast images minus pre-contrast images), axial reformatted images, and 3D maximum intensity projection (MIP) images were generated.

\section{Image analysis}

First, a retrospective analysis for the tumor location was performed by two radiologists in consensus ( $* * *$ and $* * *$ with 6 and 15 years of experience in breast MR imaging, respectively), and they were blinded to the clinicopathologic data. To determine the location of the tumor, the whole series of MR images, including axial reformatted and MIP images, were comprehensively reviewed with a picture archiving and communication system (PACS) workstation. The tumor locations were classified as follows: 1) quadrant location (upper-outer quadrant, upper-inner quadrant, lower-outer quadrant, lower-inner quadrant, and periareolar); 2) mediolateral location (medial, central, and lateral); and 3) anteroposterior location (anterior, middle, and posterior) [18]. The mediolateral and anteroposterior locations of the breast were determined based on trisection of the breast hemisphere. If a disagreement was present in determining the tumor location, the location of the tumor center was precisely identified using imaginary lines which divide the breast into thirds.

In addition, one radiologist ( $* * *$, with 6 years of experience in breast MR imaging) who was not involved in subjective determination of tumor location and was also blinded to clinicopathologic data identified the tumor location using 3D coordinates (Fig. 1) [19]. In axial MR images, the $\mathrm{x}$-axis measurement was recorded as the transverse distance from the posterior nipple line, an imaginary line running across the nipple back to and perpendicular to the pectoralis muscle. Tumors that were located lateral to the posterior nipple line were recorded as positive numbers, and those medial to the posterior nipple line were recorded as negative numbers. In sagittal MR images, the $y$-axis measurement was recorded as the anteroposterior distance from the chest wall; thus, this is equivalent to the distance from the chest wall. To account for the curvature of the chest wall, the shortest distance from the chest wall at an imaginary line that is perpendicular to the surface of the chest wall projected from the tumor was recorded. The zaxis measurement was recorded as the superoinferior distance from the posterior nipple line. 

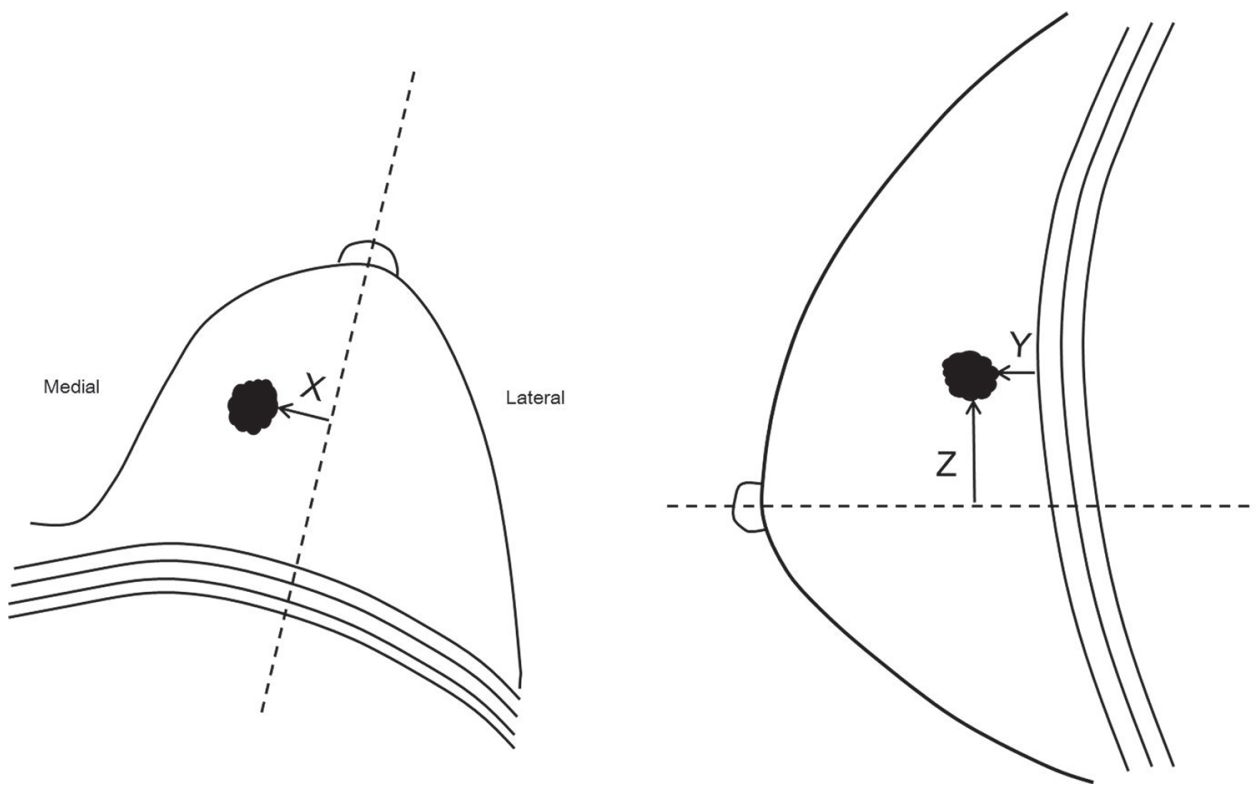

Figure 1. Diagram of tumor distances in the three-dimensional planes using breast MR images. Diagram shows how the distances in the $\mathrm{x}, \mathrm{y}$, and $\mathrm{z}$ planes were measured in axial and sagittal breast MR images. We measured the shortest distances for $\mathrm{X}$-axis $(\mathrm{X})$ and $\mathrm{z}$-axis $(\mathrm{Z})$ measurements of breast cancer in relation to the imaginary lines from the nipple and for $y$-axis $(Y)$ measurement from the chest wall.

doi:10.1371/journal.pone.0116344.g001

Tumors that were located superior to the posterior nipple line were recorded as positive numbers and those inferior to the posterior nipple line were recorded as negative numbers. As the tumors have various morphologic features including mass and non-mass enhancement, we measured the shortest distance from the margin of the lesions rather than using center of the mass as the reference points. On MR imaging, there were 1061 mass and 41 non-mass enhancement lesions.

Because each patient has a different breast size, measurements for breast size were performed in the $\mathrm{x}-, \mathrm{y}$-, and $\mathrm{z}$-axes for each patient. For the $\mathrm{x}$-axis measurement, the maximum transverse dimension of the breast size was recorded; for the y-axis measurement, the anteroposterior distance from the nipple to the chest wall was recorded; and for the z-axis measurement, the craniocaudal distance from the upper edge of the breast to the lower edge was recorded. To obtain normalized $\mathrm{x}-, \mathrm{y}^{-}$, and $\mathrm{z}$-axes distances, the recorded $\mathrm{x}-, \mathrm{y}-$, and $\mathrm{z}$-axes distances were divided by the corresponding breast size measurements. For example, the normalized y-axis distance equaled the $y$-axis distance divided by the maximum distance from the nipple to the chest wall.

\section{Histopathologic analysis}

Histopathologic diagnoses were performed by a pathologist with 25 years of experience. The histopathologic analysis included histologic type, tumor size, histologic grade (Elston and Ellis method [20], and axillary nodal status. The routinely formalin-fixed, paraffin-embedded tissue blocks were sectioned to $4 \mu \mathrm{m}$ thickness and then used for IHC. The expression of ER, PR, and HER2 was evaluated with the avidin-biotin complex IHC technique. ER and PR positivity was defined as the presence of $1 \%$ or more positively stained nuclei in ten high-power fields [21]. The HER2 expression was scored as 0 (no staining), $1+$ (weak and incomplete membrane staining), $2+$ (strong, complete membrane staining in $\leq 10 \%$ of tumor cells or weak/moderate heterogeneous complete staining in $\geq 10 \%$ of tumor cells) and $3+$ (strong, complete membrane staining in $>30 \%$ of tumor cells) by immunohistochemistry staining. Tumors with a score of 
$3+$ were classified as positive for HER2 overexpression, whereas tumors with scores of 0 or $1+$ were considered as negative. We performed FISH for tumors with a score of $2+$ using the PathVysion HER2 DNA probe (Abbott Molecular Inc., Downers Grove, IL). HER2 expression was considered positive if the ratio of HER2 gene copies to chromosome 17 signals was greater than 2.2. The ER-positive breast cancers was defined as ER-positive, HER2-negative, and PR may be positive or negative; the triple-negative subtype as ER-negative, PR-negative, and HER2-negative tumors [22].

\section{Data collection and statistical analysis}

The patient age, family history of breast cancer, palpability, mammographic density according to Breast Imaging Reporting and Data System (BI-RADS) [18], mammographic visibility, and histopathologic data were collected. To compare the clinicopathologic findings and location of the triple-negative and ER-positive breast cancers, the chi-square or Fisher's exact tests, and the unpaired Student $t$ test were used. For more than two groups of comparison, one-way analysis of variance (ANOVA) and Bonferroni-Dunn posthoc tests were performed. We used the chi-square test for trend (two sided) to assess whether a location trend existed among the histologic grades and mammographic breast densities. The independent relationship between absolute/normalized $\mathrm{x}-, \mathrm{y}-, \mathrm{z}$-axes distances and tumor subtype was assessed using multiple linear regression with age, family history of breast cancer, mammographic density, tumor size, histologic grade, and axillary nodal status as potential confounding variables. A $P$ value of less than .05 was considered to indicate statistical significance. All statistical analyses were performed using SPSS version 12.0 software (SPSS, Chicago, IL).

\section{Results}

\section{Clinicopathologic findings}

Patients with triple-negative breast cancers had more frequent family history of breast cancer $(P=.014)$, palpable symptom $(P<.0001)$, larger tumor size $(P<.0001)$, higher histologic grade $(P<.0001)$, and negative axillary nodal status $(P=.001)$. Age, mammographic breast density, and mammographic visibility of the cancers were not significantly different between patients with triple-negative and ER-positive breast cancers. Table 1 demonstrates the clinicopathologic characteristics of patients with triple-negative breast cancers and those with ER-positive breast cancers.

\section{Distribution of tumor location}

Among 1102 breast cancers, we found no significant differences in the distribution of quadrants and mediolateral locations between triple-negative and ER-positive breast cancers (Table 2). However, the distribution of the anteroposterior location was significantly different between triple-negative and ER-positive breast cancers. Triple-negative breast cancers tended to be located more posteriorly compared to ER-positive breast cancers $(P=.0026): 54.7 \%$ (140/256) of the triple-negative breast cancers had a posterior location, whereas 43.7\% (370/ 846) of the ER-positive breast cancers had a posterior location. When we defined 'prepectoral' location of tumor as the absolute distance of tumor from the chest wall less than $3 \mathrm{~mm}$, triplenegative breast cancers had more frequently prepectoral location than ER-positive breast cancers $(17 \%[43 / 256]$ vs. $11 \%[95 / 846], P=.018)$.

Patients with tumors in the medial location were significantly younger than with tumor in the central location $(P<.0001$; posthoc analysis). Patients with tumors in the posterior location were significantly younger than those with tumors in the anterior and middle locations (all $P<.0001$; posthoc analysis). Patients with denser mammographic density tended to have 
Table 1. Clinicopathologic Characteristics.

\begin{tabular}{|c|c|c|c|}
\hline Variables & Triple-Negative Cancer $(n=256)$ & ER-Positive Cancer $(n=846)$ & $P$ value \\
\hline Age $(y)^{a}$ & $51(20-78)$ & $52(22-84)$ & .069 \\
\hline Family history of breast cancer & $26(10.2)$ & $47(5.6)$ & .014 \\
\hline Palpability & & & $<.0001$ \\
\hline No & $60(23.4)$ & $379(44.8)$ & \\
\hline Yes & $196(76.6)$ & $467(55.2)$ & \\
\hline Mammographic breast density & & & $.934^{\mathrm{b}}$ \\
\hline Grade 1 & $25(9.8)$ & 75 (8.9) & \\
\hline Grade 2 & $56(21.9)$ & $185(21.9)$ & \\
\hline Grade 3 & $127(49.6)$ & $436(51.5)$ & \\
\hline Grade 4 & $48(18.8)$ & $150(17.7)$ & \\
\hline Mammographic visibility & & & .151 \\
\hline No & $19(7.4)$ & $83(9.8)$ & \\
\hline Yes & $237(92.6)$ & $763(90.2)$ & \\
\hline \multicolumn{4}{|l|}{ Histologic type } \\
\hline Invasive ductal & $235(91.8)$ & 755 (89.2) & .288 \\
\hline Invasive lobular & 0 & $45(5.3)$ & $<.0001$ \\
\hline Mucinous & 0 & $32(3.8)$ & $<.0001$ \\
\hline Metaplastic & $13(5.1)$ & 0 & $<.0001$ \\
\hline Tubular & 0 & $5(0.6)$ & .596 \\
\hline Apocrine & $4(1.6)$ & 0 & .003 \\
\hline Adenoid cystic & $2(0.8)$ & 0 & .054 \\
\hline Invasive papillary & $1(0.4)$ & $6(0.7)$ & 1.000 \\
\hline Invasive micropapillary & 0 & $2(0.2)$ & 1.000 \\
\hline Medullary & $1(0.4)$ & $1(0.1)$ & .411 \\
\hline Tumor size $(\mathrm{cm})^{\mathrm{a}}$ & $2.2(0.2-5.4)$ & $1.9(0.2-5.9)$ & $<.0001$ \\
\hline Histologic grade & & & $<.0001^{\mathrm{b}}$ \\
\hline I & $2(0.8)$ & $125(14.8)$ & \\
\hline II & $35(13.7)$ & $478(56.5)$ & \\
\hline III & $219(85.5)$ & $243(28.7)$ & \\
\hline Axillary nodal status & & & .001 \\
\hline Negative & $216(84.4)$ & $628(74.2)$ & \\
\hline Positive & $40(15.6)$ & $218(25.8)$ & \\
\hline Surgery & & & .628 \\
\hline Breast-conserving surgery & $212(82.8)$ & $712(84.2)$ & \\
\hline Mastectomy & $44(17.2)$ & $134(15.8)$ & \\
\hline
\end{tabular}

Unless otherwise indicated, data are numbers of patients and numbers in parentheses are percentages.

${ }^{a}$ Data are mean values, with ranges in parentheses.

${ }^{\mathrm{b}}$ Chi-square test for trend.

doi:10.1371/journal.pone.0116344.t001

tumors more posteriorly located $(P<.0001$; test for trend, dichotomized into posterior vs. anterior or middle). Tumors with positive axillary nodal status tended to be more laterally and anteriorly located $(P=.012$ and $P=.0085$; test for trend).

\section{Location of tumors in 3D coordinates}

Triple-negative breast cancers were significantly closer to the chest wall (absolute y-axis distance) than ER-positive breast cancers $(1.8 \mathrm{~cm}, 95 \%$ confidence interval [CI]: $1.64 \mathrm{~cm}-2.04 \mathrm{~cm}$ 
Table 2. Distribution of Tumor Location.

\begin{tabular}{llll}
\hline Variables & Triple-Negative Cancer $(\mathbf{n}=\mathbf{2 5 6})$ & ER-Positive Cancer $(\mathbf{n}=\mathbf{8 4 6})$ \\
\hline Quadrants & $126(49.2)$ & $375(44.3)$ \\
Upper outer & $73(28.5)$ & $225(26.6)$ \\
Upper inner & $26(10.2)$ & $112(13.2)$ \\
Lower outer & $18(7.0)$ & $61(7.2)$ & .174 \\
Lower inner & $13(5.1)$ & $73(8.6)$ \\
Periareolar & & & .574 \\
Mediolateral location & $51(19.9)$ & $186(22.0)$ \\
Medial & $100(39.1)$ & $310(36.6)$ \\
Central & $105(41.0)$ & $350(41.4)$ \\
Lateral & & & .064 \\
Anteroposterior & $17(6.6)$ & $30(9.5)$ \\
Anterior & $99(38.7)$ & $396(46.8)$ \\
Middle & $140(54.7)$ & $370(43.7)$ \\
Posterior & & $.0026^{\mathrm{a}}$ \\
\hline
\end{tabular}

Data are numbers of patients and numbers in parentheses are percentages

${ }^{a}$ Chi-square test for trend.

doi:10.1371/journal.pone.0116344.t002

vs. $2.3 \mathrm{~cm}, 95 \%$ CI: $2.17 \mathrm{~cm}-2.43 \mathrm{~cm} ; P<.0001$ ) (Fig. 2). The normalized y-axis distance from the chest wall was also significantly shorter for triple-negative breast cancers compared with ER-positive breast cancers ( $0.21,95 \%$ CI: $0.19-0.23$ vs. $0.25,95 \%$ CI: $0.24-0.27 ; P<.0001)$ (Table 3). The $\mathrm{x}$ - and $\mathrm{z}$-axes distances were not significantly different between triple-negative and ER-positive breast cancers. Younger patients and patients with denser mammographic density tended to have tumors that were more posteriorly located (all $P<.0001$ ) (Tables A and $B$ in S1 File). Tumors with higher histologic grade also tended to be more posteriorly located ( $P<.0001$ and $P=.0002$ for absolute and normalized $y$-axes distances). Tumors with negative axillary nodal status tended to be more posteriorly located $(P=.007$ and $P=.002$ for absolute and normalized y-axes distances).

Multiple linear regression analysis revealed that age, mammographic density, axillary nodal status, and triple-negative subtype were significantly correlated with both the absolute and normalized y-axis distances from the chest wall (all $P<.05)$ (Table 4).

\section{Discussion}

In this study, our hypothesis was that the location of triple-negative breast cancers may have a tendency to be located in the posterior or medial region of the breast, since some of the tumors seen in high risk patients or patients with BRCA-1 gene previously reported posterior position and triple-negative breast cancers have been reported to have less axillary lymph node metastasis but poorer prognosis due to distant metastasis compared with ER-positive cancers. Our results show that triple-negative breast cancers have a tendency toward posterior and prepectoral locations on MR imaging compared to ER-positive breast cancers. The frequent association of triple-negative breast cancers with a posterior or prepectoral location and rapid tumor growth rate may explain why triple-negative breast cancers often present as interval cancers on mammography [23]. There was, however, no difference in the distribution of quadrants and mediolateral locations between triple-negative and ER-positive breast cancers. We also found that age, mammographic breast density, and axillary nodal status are associated with tumor 


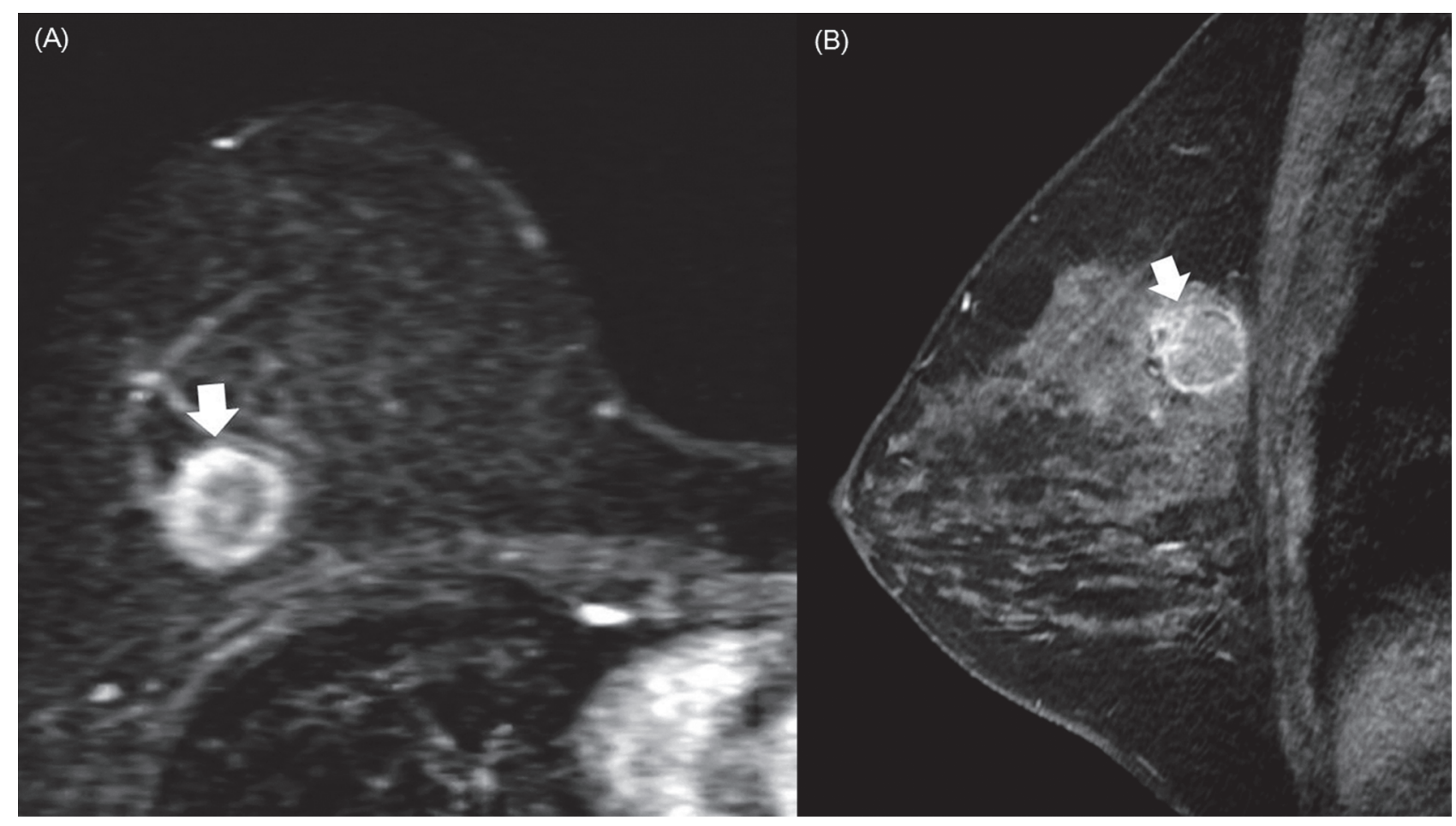

Figure 2. A triple-negative breast cancer in the posterior location. A triple-negative breast cancer in the posterior location of the right breast in a 41 -yearold woman. (A) Sagittal early contrast-enhanced nonsubtracted T1-weighted MR image with fat suppression shows a $1.5 \mathrm{~cm}$ round mass (arrow) with rim enhancement. Note the tumor location in the immediate prepectoral region. (B) Mass (arrow) is also seen on subtracted axial reformatted image.

doi:10.1371/journal.pone.0116344.g002

location. Younger patients and patients with denser mammographic density tended to have tumors that were more posteriorly located (all $P<.0001$ ).

Schrading and Kuhl investigated imaging features in women with familial risk and found that the imaging phenotypes of cancers differ among risk categories [6]. Compared with breast cancers in women with moderate familial risk, breast cancers in women with high familial risk and BRCA1 mutation carriers often exhibit benign morphologic features (noncalcified mass with an oval shape and smooth margin) and were located in the posterior or prepectoral region of the breast. However, these authors did not analyze the results according to tumor subtype. Our results may account for their observation that BRCA1-associated breast cancers more

Table 3. Location of Tumors in 3D Coordinates.

\begin{tabular}{lll}
\hline Parameters & Triple-Negative Cancer $(\mathbf{n}=\mathbf{2 5 6})$ & ER-Positive Cancer $(\mathbf{n}=\mathbf{8 4 6})$ \\
\hline $\begin{array}{l}\text { Distance }(\mathrm{cm}) \\
\text { X-axis distance from the posterior nipple line }\end{array}$ & $0.5(0.31,0.68)$ & $\boldsymbol{P}$ value \\
$\begin{array}{l}\text { Y-axis distance from the chest wall } \\
\text { Z-axis distance from the posterior nipple line }\end{array}$ & $1.8(1.64,2.04)$ & $0.4(0.33,0.53)$ \\
Normalized distance & $1.2(0.92,1.44)$ & $2.3(2.17,2.43)$ \\
X-axis distance from the posterior nipple line & & $0.9(0.78,1.05)$ \\
Y-axis distance from the chest wall & $0.06(0.04,0.08)$ & .528 \\
Z-axis distance from the posterior nipple line & $0.21(0.19,0.23)$ & $0.05(0.04,0.07)$ \\
\hline
\end{tabular}

Data are mean values, with $95 \%$ confidence intervals in parentheses.

${ }^{a}$ Ranges are (-0.5-0.5) for X-and Z-axes distances from the posterior nipple line and (0-1.0) for Y-axis distance from the chest wall.

doi:10.1371/journal.pone.0116344.t003 
Table 4. Multiple Linear Regression Analysis of Association of Variables with Distance from the Chest Wall.

\begin{tabular}{llll}
\hline Parameters & \multicolumn{2}{c}{ Unstandardized Coefficients } & Standardized $\beta$ Coefficient \\
\cline { 2 - 4 } & $\boldsymbol{\beta}$ & Standard Error & \\
\hline Absolute y-axis distance & & & \\
Age & 0.027 & 0.007 & 0.150 \\
Family history of breast cancer & 0.203 & 0.250 & 0.024 \\
Mammographic density & -0.511 & 0.082 & -0.228 \\
Triple-negative breast cancer & -0.167 & 0.075 & -0.073 \\
Tumor size & -0.062 & 0.045 & -0.042 \\
Histologic grade & -0.085 & 0.070 & -0.039 \\
Axillary nodal status & 0.376 & 0.137 & 0.085 \\
Normalized y-axis distance & & & \\
Age & 0.002 & 0.001 & .0001 \\
Family history of breast cancer & 0.020 & 0.024 & 0.122 \\
Mammographic density & -0.038 & 0.008 & 0.025 \\
Triple-negative breast cancer & -0.016 & 0.007 & -0.175 \\
Tumor size & -0.009 & 0.004 & -0.073 \\
Histologic grade & -0.009 & 0.007 & -0.061 \\
Axillary nodal status & 0.047 & 0.013 & -0.046 \\
\hline
\end{tabular}

doi:10.1371/journal.pone.0116344.t004

frequently had a posterior location because BRCA1 mutation carriers have a greater tendency to have triple-negative cancers $[24,25]$.

Most published research regarding tumor location has utilized mammograms or clinical examinations, and has assessed the two-dimensional (2D) plane with respect to quadrants or mediolateral location [1-5]. Tumor location has rarely been investigated using the sagittal plane and anteroposterior location $[6,26]$. Compared with mammography, MR imaging has many advantages for assessing the tumor location in a breast including imaging without compression, multiplanar imaging planes, and the ability to image the chest wall [27]. In addition, the sensitivity of MR imaging is higher than that of mammography, particularly in women with dense breasts [28]. These advantages are especially important when identifying the location of small cancers in the prepectoral region. To the best of our knowledge, this study is the largest series of patients in whom the location of breast cancers was assessed using MR imaging.

In our study, axillary nodal involvement was found to be less frequent in the tumors located posteriorly. As we mentioned earlier, tumors in the posterior location tended to have more non-axillary lymph node pathway than tumors in the anterior location [17]. A tendency toward posterior location in triple-negative cancers may explain that alleged clinical paradox of triple-negative breast cancers having weak relationship between tumor size, axillary nodal status, and survival $[29,30]$. Our finding suggests that posterior tumor location in the triplenegative cancers might be a high risk feature for non-axillary lymph node pathway, which may need an adjuvant therapy including systemic therapy.

Our study has several limitations. First, we did not analyze all of the intrinsic subtypes of breast cancers including luminal, luminal/HER2, HER2-enriched, and basal-like subtypes [22, 31]. The definition and classification of molecular subtypes based on IHC profiles are still controversial [32]. In this study, we attempted to demonstrate an asymmetrical distribution of location according to breast cancer subtypes by comparing triple-negative and ER-positive breast cancers, which are known to be the most contrasting breast cancer subtypes. Comparison with other intrinsic subtype including luminal/HER2, HER2-enriched needs to be investigated for 
further research. Second, we did not analyze the results according to the triple-negative breast cancer subtype or BRCA1 mutation status. Triple-negative breast cancer is a heterogeneous disease, and the basal-like, mesenchymal-like, immune modulatory, and luminal androgen receptor subgroups have been reported [33]. Further studies are warranted to explore the impact of tumor location on the prognosis of women with triple-negative breast cancers. Third, we used two different parameters to determine tumor location. In our subjective analysis, we determined tumor location by the radiologists estimating the center of the tumor, which is similar to the clinical practice. However, using the tumor center in the objective measurement, the distance of the tumor from the reference point would be affected by the tumor size; larger tumors, particularly in prepectoral location, tend to be measured with larger distance from the chest wall. Thus, we used the margin of the tumor rather than the center of the tumor to measure the distance from the reference points. In addition, intra-observer reproducibility test for 3D coordinates in identifying tumor location was not performed in our study. However, most of our study cases were masses (96.3\%) and have discrete margins.

Lastly, we did not provide mechanisms for the skewed location toward the posterior and prepectoral breast in triple-negative breast cancers. Hypothetical explanation for our observation is that posterior or prepectoral location may have a favorable environment for developing triple-negative breast cancers. The idea that microenvironment shapes the course of carcinogenesis, and hence breast cancer subtype has been discussed [34,35]. It is interesting to note that age and mammographic density are also associated with posterior location of the tumor in our study. In various tumors, a correlation between distinctive tumor location and genetic signatures or clinical behaviors has been reported [36-38]. Further biological and radiogenomic studies are needed to explain the relationship between distinctive locations and breast cancer subtypes.

In conclusion, triple-negative breast cancers have a tendency toward a posterior or prepectoral location on MR imaging compared with ER-positive breast cancers. Special attention should be paid to these regions of the breast to detect biologically aggressive triplenegative breast cancers.

\section{Supporting Information}

S1 File. This file contains supporting Tables A and B. Table A. Distances of Tumors in 3D Coordinates According to the Clinicopathologic Features

Table B. Normalized Distances of Tumors in 3D Coordinates According to the Clinicopathologic Features

(DOC)

\section{Author Contributions}

Conceived and designed the experiments: WHK WKM. Performed the experiments: WHK WKM JMC. Analyzed the data: WHK NC. Contributed reagents/materials/analysis tools: WHK WH IAP. Wrote the paper: WHK NC WKM.

\section{References}

1. Zlatescu MC, TehraniYazdi A, Sasaki H, Megyesi JF, Betensky RA, et al. (2001) Tumor location and growth pattern correlate with genetic signature in oligodendroglial neoplasms. Cancer Res 61:67136715. PMID: 11559541

2. Colleoni M, Zahrieh D, Gelber RD, Holmberg SB, Mattsson JE, et al. (2005) Site of primary tumor has a prognostic role in operable breast cancer: the international breast cancer study group experience. $J$ Clin Oncol 23: 1390-1400. PMID: 15735115 
3. Lohrisch C, Jackson J, Jones A, Mates D, Olivotto IA (2000) Relationship between tumor location and relapse in 6,781 women with early invasive breast cancer. J Clin Oncol 18: 2828-2835. PMID: 10920130

4. Manjer J, Balldin G, Garne JP (2004) Tumour location and axillary lymph node involvement in breast cancer: a series of 3472 cases from Sweden. Eur J Surg Oncol 30: 610-617. PMID: 15256233

5. Yoshihara E, Smeets A, Laenen A, Reynders A, Soens J, et al. (2012) Predictors of axillary lymph node metastases in early breast cancer and their applicability in clinical practice. Breast.

6. Schrading S, Kuhl CK (2008) Mammographic, US, and MR imaging phenotypes of familial breast cancer. Radiology 246: 58-70. PMID: 18096529

7. Wolfe JN (1974) Analysis of 462 breast carcinomas. Am J Roentgenol Radium Ther Nucl Med 121: 846-853. PMID: 4432966

8. Bird RE, Wallace TW, Yankaskas BC (1992) Analysis of cancers missed at screening mammography. Radiology 184: 613-617. PMID: 1509041

9. Harvey JA, Nicholson BT, Cohen MA (2008) Finding early invasive breast cancers: a practical approach. Radiology 248: 61-76. doi: 10.1148/radiol.2481060339 PMID: 18566169

10. Curigliano G, Goldhirsch A (2011) The triple-negative subtype: new ideas for the poorest prognosis breast cancer. J Natl Cancer Inst Monogr 2011: 108-110. doi: 10.1093/jncimonographs/lgr038 PMID: 22043054

11. Foulkes WD, Smith IE, Reis-Filho JS (2010) Triple-negative breast cancer. N Engl J Med 363: 19381948. doi: 10.1056/NEJMra1001389 PMID: 21067385

12. Carey L, Winer E, Viale G, Cameron D, Gianni L (2010) Triple-negative breast cancer: disease entity or title of convenience? Nat Rev Clin Oncol 7: 683-692. PMID: 20877296

13. Crabb SJ, Cheang MC, Leung S, Immonen T, Nielsen TO, et al. (2008) Basal breast cancer molecular subtype predicts for lower incidence of axillary lymph node metastases in primary breast cancer. Clin Breast Cancer 8: 249-256. doi: 10.3816/CBC.2008.n.028 PMID: 18650155

14. Uematsu T, Kasami M, Yuen S (2009) Triple-negative breast cancer: correlation between MR imaging and pathologic findings. Radiology 250: 638-647. PMID: 19244039

15. Dogan BE, Turnbull LW (2012) Imaging of triple-negative breast cancer. Ann Oncol 23 Suppl 6: vi2329. PMID: 23012298

16. Krizmanich-Conniff KM, Paramagul C, Patterson SK, Helvie MA, Roubidoux MA, et al. (2012) Triple receptor-negative breast cancer: imaging and clinical characteristics. AJR Am J Roentgenol 199: 458464. doi: 10.2214/AJR.10.6096 PMID: 22826413

17. Tanis PJ, van Rijk MC, Nieweg OE (2005) The posterior lymphatic network of the breast rediscovered. J Surg Oncol 91: 195-198. PMID: 16118774

18. D'Orsi CJ, Hall FM (2006) BI-RADS lexicon reemphasized. AJR Am J Roentgenol 187: W557; discussion W558; author reply W559.PMID: 17056895

19. Sandhu PS, Joe BN, Coakley FV, Qayyum A, Webb EM, et al. (2007) Bowel transition points: multiplicity and posterior location at CT are associated with small-bowel volvulus. Radiology 245: 160-167. PMID: 17717325

20. Elston CW, Ellis IO (1991) Pathological prognostic factors in breast cancer. I. The value of histological grade in breast cancer: experience from a large study with long-term follow-up. Histopathology 19: 403-410. PMID: 12405947

21. Hammond ME, Hayes DF, Dowsett M, Allred DC, Hagerty KL, et al. (2010) American Society of Clinical Oncology/College Of American Pathologists guideline recommendations for immunohistochemical testing of estrogen and progesterone receptors in breast cancer. J Clin Oncol 28: 2784-2795. doi: 10. 1200/JOP.777003 PMID: 21037871

22. Cancer Genome Atlas N (2012) Comprehensive molecular portraits of human breast tumours. Nature 490: 61-70. doi: 10.1038/nature11412 PMID: 23000897

23. Collett K, Stefansson IM, Eide J, Braaten A, Wang H, et al. (2005) A basal epithelial phenotype is more frequent in interval breast cancers compared with screen detected tumors. Cancer Epidemiol Biomarkers Prev 14: 1108-1112. PMID: 15894660

24. Atchley DP, Albarracin CT, Lopez A, Valero V, Amos Cl, et al. (2008) Clinical and pathologic characteristics of patients with BRCA-positive and BRCA-negative breast cancer. J Clin Oncol 26: 4282-4288. PMID: 18779615

25. Lee E, McKean-Cowdin R, Ma H, Spicer DV, Van Den Berg D, et al. (2011) Characteristics of triple-negative breast cancer in patients with a BRCA1 mutation: results from a population-based study of young women. J Clin Oncol 29: 4373-4380. doi: 10.1200/JCO.2010.33.6446 PMID: 22010008 
26. Guth U, Wight E, Langer I, Schotzau A, Dieterich H, et al. (2006) Breast cancer sagittal/horizontal plane location influences axillary lymph node involvement. Eur J Surg Oncol 32: 287-291. PMID: 16466903

27. Morris EA, Schwartz LH, Drotman MB, Kim SJ, Tan LK, et al. (2000) Evaluation of pectoralis major muscle in patients with posterior breast tumors on breast MR images: early experience. Radiology 214: 67-72. PMID: 10644103

28. Bluemke DA, Gatsonis CA, Chen MH, DeAngelis GA, DeBruhl N, et al. (2004) Magnetic resonance imaging of the breast prior to biopsy. JAMA 292: 2735-2742. PMID: 15585733

29. Dent R, Trudeau M, Pritchard KI, Hanna WM, Kahn HK, et al. (2007) Triple-negative breast cancer: clinical features and patterns of recurrence. Clin Cancer Res 13:4429-4434. PMID: 17671126

30. Ugras S, Stempel M, Patil S, Morrow M (2014) Estrogen Receptor, Progesterone Receptor, and HER2 Status Predict Lymphovascular Invasion and Lymph Node Involvement. Ann Surg Oncol 21:37803786. PMID: 24952028

31. Perou CM, Sorlie T, Eisen MB, van de Rijn M, Jeffrey SS, et al. (2000) Molecular portraits of human breast tumours. Nature 406: 747-752. PMID: 10963602

32. Prat A, Cheang MC, Martin M, Parker JS, Carrasco E, et al. (2013) Prognostic significance of progesterone receptor-positive tumor cells within immunohistochemically defined luminal a breast cancer. J Clin Oncol 31: 203-209. doi: 10.1200/JCO.2012.43.4134 PMID: 23233704

33. Lehmann BD, Bauer JA, Chen X, Sanders ME, Chakravarthy AB, et al. (2011) Identification of human triple-negative breast cancer subtypes and preclinical models for selection of targeted therapies. J Clin Invest 121: 2750-2767. doi: 10.1172/JCI45014 PMID: 21633166

34. Barcellos-Hoff MH (2013) Does microenvironment contribute to the etiology of estrogen receptor-negative breast cancer? Clin Cancer Res 19: 541-548. PMID: 23325583

35. Dang TT, Prechtl AM, Pearson GW (2011) Breast cancer subtype-specific interactions with the microenvironment dictate mechanisms of invasion. Cancer Res 71: 6857-6866. doi: 10.1158/0008-5472. CAN-11-1818 PMID: 21908556

36. Gilbertson RJ, Gutmann DH (2007) Tumorigenesis in the brain: location, location, location. Cancer Res 67: 5579-5582. PMID: 17575119

37. Slattery ML, Wolff E, Hoffman MD, Pellatt DF, Milash B, et al. (2011) MicroRNAs and colon and rectal cancer: differential expression by tumor location and subtype. Genes Chromosomes Cancer 50: 196206. doi: 10.1002/gcc.20844 PMID: 21213373

38. Ellingson BM, Lai A, Harris RJ, Selfridge JM, Yong WH, et al. (2013) Probabilistic radiographic atlas of glioblastoma phenotypes. AJNR Am J Neuroradiol 34: 533-540. doi: 10.3174/ajnr.A3253 PMID: 22997168 